
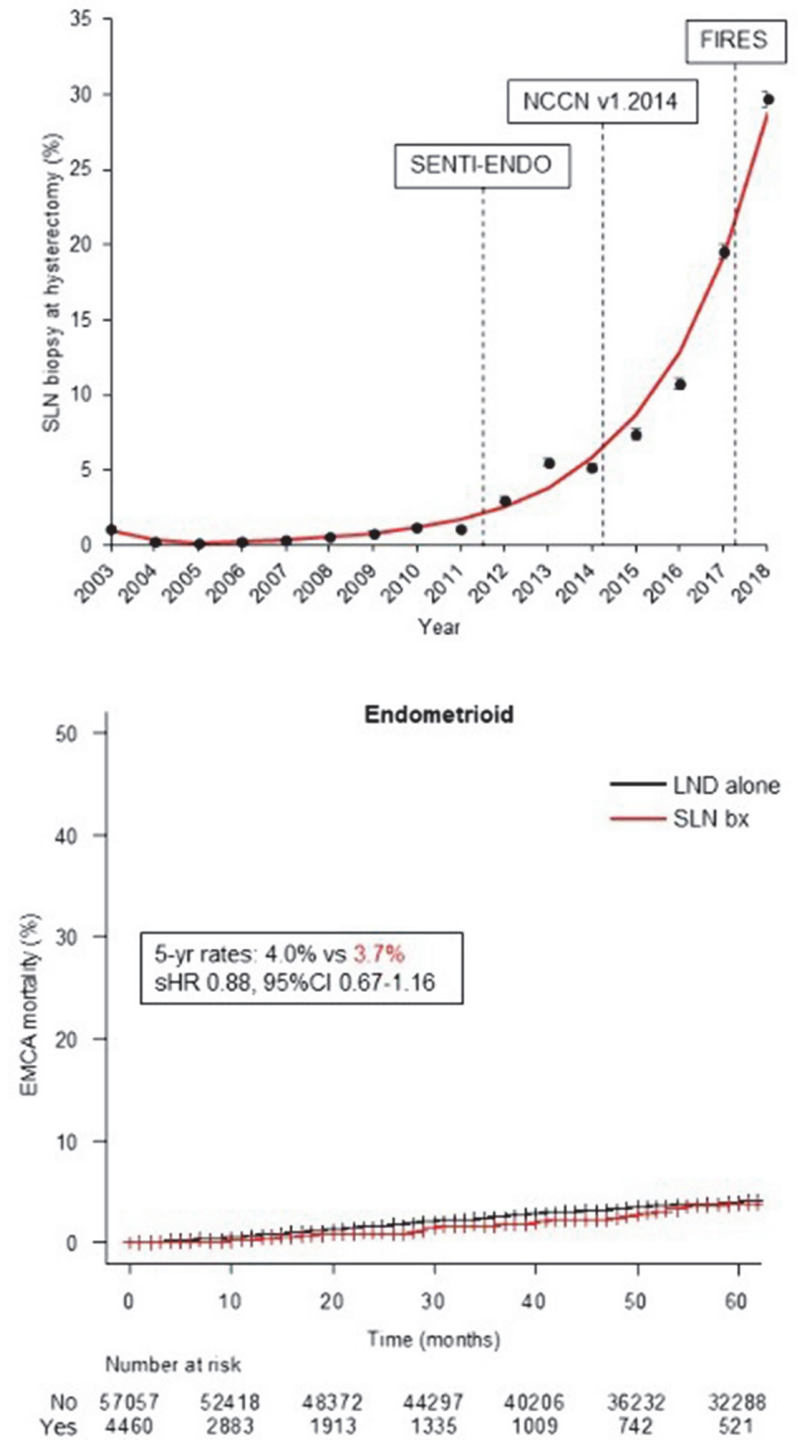

Abstract EPV093b/\#769 Figure 1

HR 0.96, 95\%CI 0.82-1.1; bottom-panel) and non-endometriod histology (subdistribution-HR 0.85 , 95\%CI 0.69-1.0) in propensity score weighted models. In low-risk endometrial cancer, the increase in recent SLN biopsy resulted in 15.3 percent point increase in the surgical nodal evaluation by 2018 (expected versus observed rates, 37.8\% versus 53.1\%).

Conclusions The landscape of surgical nodal evaluation is shifting from lymphadenectomy to SLN biopsy in early endometrial cancer. Effects of SLN biopsy-based surgical treatment on endometrial cancer survival warrants further confirmation.

\section{EPV094/\#103 MALIGNANT PERIVASCULAR EPITHELIOID TUMOR OF THE UTERUS ASSOCIATED WITH HYPERPROLACTINEMIA}

JI Argel*, D Benavides. Philippine General Hospital, Obstetrics and Gynecology, Manila, Philippines

10.1136/ijgc-2021-IGCS. 164
Objectives Malignant perivascular epitheliold tumors (PEComas) are rare mesenchymal tumors originating from perivascular epithelioid cells with specific histologic and immunologic features. Due to its rarity, lack of specific clinical findings, aggressive and unpredictable biologic behavior, this type of tumor is difficult to manage and there is no standard therapeutic strategy.

Methods A 34-year-old G1P1(1001) presented with a history of galactorrhea (elevated prolactin $313 \mathrm{ng} / \mathrm{mL}$ ) and irregular menstruation. On work-up, cranial magnetic resonance imaging (MRI) revealed no mass on the pituitary gland, abdominopelvic MRI showed a large uterine mass. She was initially treated medically which offered no relief of symptoms. She was then diagnosed and managed as a case of ectopic prolactin secreting leiomyoma uteri. Myomectomy was performed and prolactin level decreased to normal level $(6.3 \mathrm{ng} / \mathrm{mL})$ and with resolution of symptoms. Histopathology revealed malignant PEComa. Prolactin increased when tumor recurred and she underwent re-exploration and tumor debulking. Specimen from first and second operation were compared and shared the same histomorphological features. Immunohistochemical stain for prolactin was performed because of the suspicion of ectopic prolactin secreting tumor but revealed a negative result. The patient was given 3 cycles of Doxorubicin.

Results Endocrine paraneoplastic syndrome is the production of hormonal substances that produce unique clinical syndromes, example is prolactin. Ectopic prolactin secretion is the production of hormone by a cell type that does not normally produce the hormonal substance or produces it normally at very low levels.

Conclusions The index case showed malignant PEComa of the uterus associated with hyperprolactinemia with negative immunohistochemical stain for prolactin.

\section{EPV095/\#104 EXAMINING THE RISK OF COLORECTAL CANCER IN PATIENTS WITH MLH-1 PROMOTER HYPERMETHYLATED ENDOMETRIAL CANCER}

A Kanbergs*, L Philp, K James, T Randall. Massachusetts General Hospital, Obstetrics and Gynecology, Boston, USA

\subsection{6/ijgc-2021-IGCS. 165}

Objectives DNA Microsatellite instability (MSI) due to hypermethylation of the MLH1 gene leading to deficient DNA mismatch repair (MMR) is a frequent finding in sporadic endometrial (EC) and colorectal cancers (CRC). Individuals with germline MMR mutations have an $80 \%$ lifetime risk of colorectal cancer (CRC) and follow strict cancer screening protocols. It is unclear if women found to have sporadic MSI high EC have an increased risk of colorectal malignancy. The objective of this study was to determine if there is an increased risk of CRC in patients with MLH-1 promoter hypermethylated EC as compared to patients with microsatellite stable (MSS) disease.

Methods We performed a retrospective cohort study of all cases of EC with known MMR status treated at Massachusetts General Hospital between 2013-2019. Patients with germline MMR mutations were excluded. ICD-9/10 codes from electronic medical records were used to determine the incidence 
of CRC in the two groups. Chi-squared testing was used to assess for differences in the proportion of CRC between MMR groups with $\mathrm{p}<0.05$ considered significant.

Results Among 988 patients with EC not associated with a germline MMR mutation, 16\% $(\mathrm{n}=162)$ had MLH-1 promotor hypermethylation and $84 \%(n=826)$ did not. Among those with MLH-1 promotor hypermethylation there were 6 cases (3.6\%) of CRC vs. 34 cases $(4.1 \%)$ in those with MSS disease $(\mathrm{p}=.743)$.

Conclusions We found no difference in incidence of CRC in individuals with MLH-1 promotor hypermethylated EC as compared with those with MSS disease. Patients with MLH-1 promotor hypermethylated EC should follow general CRC screening guidelines.

\section{EPV096/\#110 LSR ACTIVATES MAPK PATHWAY AND PROMOTES CELL PROLIFERATION AND INVASION IN ENDOMETRIAL CANCER: ANALYSIS OF BIOINFORMATICS-BASED SIGNAL TRANSDUCTION}

${ }^{1} Y$ Nagase*, ${ }^{1} \mathrm{~K}$ Hiramatsu, 'S Nakagawa, 'S Matsuzaki, 'T Kimura, ${ }^{2} \mathrm{~S}$ Serada, ${ }^{1} Y$ Ueda, ${ }^{2} \mathrm{~T}$ Naka, ${ }^{1} \mathrm{~T}$ Kimura. ${ }^{1}$ Osaka University Graduate School of Medicine, Department of Obstetrics and Gynecology, Suita, Osaka, Japan; ${ }^{2}$ Kochi University, Department of Clinical Immunology, Nankoku, Kochi, Japan

\subsection{6/ijgc-2021-IGCS.166}

Objectives Lipolysis-stimulated lipoprotein receptor (LSR) is a membrane protein that has been studied in various malignant tumors. We previously reported that high expression of LSR was associated with poor prognosis, advanced stage, deep myometrial invasion, and metastasis in endometrial cancer (EC). However, the mechanism by which LSR affects patient's prognosis remains largely unclear. Here, we aimed to investigate the functions of LSR in EC.

Methods Cell proliferation and invasion were analyzed using LSR-knockdown cell lines (HEC1 and HEC116), and the activity of several signaling pathways were examined by Western blotting. To investigate the function of LSR in EC cells, the pathway enrichment and ontology analysis were performed using the publicly available proteomic data.

Results LSR-knockdown significantly suppressed cell proliferation in WST-8 assay. The pathway analysis demonstrated that MAPK signaling pathway was enriched in proteins correlated with high LSR expression. In ontology analysis, we found several biological processes, including 'regulation of ERK1/2' and 'MAPK cascade.' Following the results of pathway enrichment and ontology analysis, we confirmed that LSR-knockdown downregulated the phosphorylation of MEK/ERK pathway, including MEK1/2, ERK1/2, and p90RSK in western blotting. Cell invasion assay and western blot analysis demonstrated that LSR-knockdown suppressed MT1-MMP/MMP2 expression and cell invasion. Interestingly, ERK1/2-knockdown also suppressed MT1-MMP/MMP2 expression, suggesting that LSR activated MT1-MMP/MMP2 via ERK1/2 and promoted cell invasion.

Conclusions Our results of in vitro study and bioinformatic analysis showed that LSR regulated cell proliferation and invasion via $\mathrm{MEK} / \mathrm{ERK}$ pathway, and contributed poor prognosis in EC. LSR may be a new therapeutic target of advanced EC.

\section{EPV097/\#140 APPLICATION OF A MACHINE LEARNING ALGORITHM TO IDENTIFY PREDICTORS OF RECURRENCE AND RECURRENCE FREE SURVIVAL IN HIGH GRADE ENDOMETRIAL CANCER}

${ }^{1} \mathrm{~S}$ Piedimonte*, ${ }^{2} \mathrm{~T}$ Feigenberg, ${ }^{3} \mathrm{~B}$ Cormier, ${ }^{4} \mathrm{~J}$ Kwon, ${ }^{5} \mathrm{~W}$ Gotlieb, ${ }^{6} \mathrm{M}$ Plante, ${ }^{5} \mathrm{~S}$ Lau ${ }^{7} \mathrm{~L}$ Helpman, ${ }^{6} \mathrm{MC}$ Renaud, ${ }^{8} \mathrm{~T}$ May, ${ }^{9} \mathrm{D}$ Vicus. ' University of Toronto, Gynecologic Oncolgoy, Toronto, Canada; ${ }^{2}$ Trillium Health Partners, Gynecologic Oncolgoy, missassauga, Canada; ${ }^{3}$ Centre hospitalier de I'Université de Montréal, Gynecologic Oncology, Montreal, Canada; ${ }^{4}$ Vancouver General Hospital, Gynecologic Oncolgoy, Vancouver, Canada; ${ }^{5}$ McGill University, Jewish General Hospital, Gynecology Oncology, Montreal, Canada; ${ }^{6}$ Hotel Dieu de Quebec, Gynecology Oncology, Quebec, Canada; ${ }^{7} J u r a v i n s k i$ Cancer Center, Gynecologic Oncology, Hamilton, Canada; ${ }^{8}$ Princess Margaret Cancer Centre/University of Health Network/Sinai Health Systems, Gynecologic Oncology, Toronto, Canada; ${ }^{9}$ Sunnybrook Health Sciences Centre, Gynecologic Oncology, Toronto, Canada

\subsection{6/ijgc-2021-IGCS.167}

Objectives To train various machine learning algorithms to predict recurrence and recurrence-free survival (RFS) in highgrade endometrial cancer (HGEC)

Methods Data was retrospectively collected across 8 Canadian centers including 1237 patients and divided arbitrarily 50\% training, 25\% validation and 25\% testing. Four models were trained to predict recurrence: random forests, boosted trees, and 2 neural networks. Receiver operating characteristic curves (ROC) were used to determine model performance and select the best model based on highest area under the curve (AUC) in the test set. For time to recurrence models, we trained a random forest and Lasso model compared to Cox Proportional hazards. Concordance was reported using a c-statistic.

Results Among the 4 models tested, the bootstrap random forest had the best AUC in the test set and was the best model to predict recurrence in HGEC; the AUCs were $85.2 \%, 74.1 \%$ and $71.8 \%$ in the training, validation and test sets respectively. The top 5 predictors were: stage, uterus height, specimen weight, adjuvant chemotherapy and pre-operative histology. When stratified by stage, the AUC in the test set increased to $77 \%$ for Stage III and $80 \%$ for Stage IV. For time to recurrence, there was no difference between the Lasso and Cox Proportional Hazards models (test set c-index 71\%) while the random forest had a cindex of $60.5 \%$.

Conclusions A bootstrap random forest model best predicted recurrence in HGEC; model prediction further improved in Stage III and IV patients. Machine learning survival models performed similar to Cox Proportional Hazards but could be conducted with greater efficiency.

\section{EPV098/\#177 PROSPECTS FOR IMPROVING THE METHODS OF COMPLEX TREATMENT OF PATIENTS WITH ENDOMETRIAL CANCER STAGE I}

O Movchan*, V Svintsitskiy, O Renkas. National Cancer Institute, Oncogynecology, Kiyv, Ukraine

\subsection{6/ijgc-2021-IGCS. 168}

Objectives The analysis was performed in 968 women with endometrioid stage I endometrial cancer who underwent hysterectomy without/with adjuvant therapy (radiation or chemotherapy) in the Oncologynecology Research Department of the 